CLINICAL STUDY

\title{
Can we ever stop imaging in surgically treated and radiotherapy- naive patients with non-functioning pituitary adenoma?
}

\author{
Raghava Reddy, Simon Cudlip ${ }^{1}$, James V Byrne², Niki Karavitaki and John A H Wass \\ Department of Endocrinology, Oxford Centre for Diabetes, Endocrinology and Metabolism, Churchill Hospital, University of Oxford, Oxford OX3 7LJ, UK, \\ Departments of ${ }^{1}$ Neurosurgery and ${ }^{2}$ Neuroradiology, The John Radcliffe Hospital, Oxford, UK \\ (Correspondence should be addressed to J A H Wass; Email: john.wass@noc.nhs.uk)
}

\begin{abstract}
Background: Non-functioning pituitary adenomas (NFAs) are slow-growing tumours with reported re-growth rates following surgical resection alone of up to $50 \%$ at 10 years. Currently, the desired length of follow-up surveillance imaging in un-irradiated patients is unclear.

Aim: To clarify the timing of re-growth in patients with NFAs, treated solely by surgery without post-operative pituitary radiotherapy, and also to clarify whether continued imaging is necessary in these patients.

Methods: A case note analysis of all patients who underwent surgery alone for NFA between January 1984 and December 2007 was undertaken. Patients were followed for a minimum of 1 year. Re-growth was diagnosed on the basis of radiological appearances with or without associated manifestations.

Results: One hundred and fifty-five patients (94 males, mean age at diagnosis 57.9 (range 18.3-88) years) were included. Twenty-nine were followed up for more than 10 years. The mean follow-up following surgery was 6.1 years (median 4.3 (range 1-25.8)). Re-growth was documented in 54 $(34.8 \%)$ cases and $20.4 \%$ of these cases showed relapse/re-growth 10 or more years after the initial surgery. Kaplan-Meier analysis showed relapse rates of 23.1, 46.7 and $67.9 \%$ at 5, 10 and 15 years respectively. There was a significant increase in the re-growth rates if there was either pituitary tumour remnant observed on the first post-operative scan $(P \leq 0.001)$ or a younger age at initial surgery $(P=0.034)$.

Conclusion: These results suggest that patients with NFAs need to be closely monitored following surgery, particularly those with post-operative tumour remnants. With $20 \%$ of relapse occurring after 10 years, follow-up surveillance needs to be continued beyond this time.

European Journal of Endocrinology 165 739-744
\end{abstract}

\section{Introduction}

Clinically non-functioning pituitary adenomas (NFAs) are the second commonest type of pituitary adenoma, comprising $\sim 14.7 \%$ of pituitary tumours (1). They have a prevalence of 70-90 cases/million population (2). They are benign and slow growing and are often large at the time of diagnosis. Owing to their critical location, these tumours commonly present with mass effects including headaches, visual field defects and hypopituitarism. Occasionally, they are detected incidentally on head scans performed to investigate other cranial conditions, and our studies report a significant increase in their size during follow-up if these are not operated on (3). Surgery, usually via the transsphenoidal route, forms the main stay of treatment for macroadenomas already causing pressure symptoms and also for those deemed to threaten the vision in the near future due to their close proximity to optic chiasm. With surgery alone, studies have reported a re-growth rate of up to $30 \%$ at 5 years and $50 \%$ at 10 years (4-13). Hence, pituitary radiotherapy (RT) is frequently used as adjuvant therapy following surgery, due to its efficacy in preventing the re-growth of NFA remnants following initial surgical debulking (13-17), particularly in patients with unresectable tumour, for example in the cavernous sinus. However, the indications for using pituitary RT remain controversial, as not all tumour remnants will re-grow and there are concerns relating to potential complications following this treatment (14-16) including pituitary hormone deficiencies, late optic chiasmal damage, late memory impairment and late second tumour formation.

In Oxford, it has been our practice to follow nonfunctioning tumours by serial imaging yearly for the first 5 years and 2 years thereafter unless the tumour involves the cavernous sinus to a considerable degree or has histological features of aggressive tumour behaviour (e.g. mitosis or poor differentiation), in which case immediate post-operative radiation is considered. 
At present, there are no reliable clinical, biochemical, radiological or pathological parameters that predict the likelihood of late tumour re-growth. Therefore, patients who are managed by surgery alone and not followed by RT treatment need long-term follow-up with regular magnetic resonance imaging (MRI; computed tomography (CT) if contraindicated or unavailable) of the pituitary to detect disease relapse. However, the desired duration of follow-up imaging is unclear. We have previously reported re-growth rates in unirradiated non-functioning tumours at 10 years (7), but long-term follow-up data using a fixed imaging protocol are lacking. We reasoned that all re-growths might occur within a certain time frame. For sometime, we have had a policy of regular surveillance scans but wished to assess the time frame for which follow-up imaging was necessary.

\section{Patients and methods}

We assessed retrospectively the case notes and scans of all pituitary NFA patients treated surgically in our department in Oxford between January 1984 and December 2007. A diagnosis of NFA (including silent ACTH) was made on the basis of lack of clinical and biochemical evidence of hormone excess in addition to histological and immunohistochemical evaluation. The inclusion criteria for this study were as follows: i) the diagnosis of NFA, ii) primary treatment with surgery and iii) a minimum of 1-year post-operative follow-up with a pituitary scan. If a patient received RT more than a year after the operation, any scans before RT were included in the study.

Follow-up management included regular clinical evaluation and imaging at regular intervals. Pituitary tumours were monitored using MRI after 1993 in all patients; CT was used before $1993(n=12)$ or if there was a contraindication to MRI $(n=5)$. A standard protocol of T2-weighted axial and T1-weighted coronal and sagittal high-resolution spin-echo sequences were performed. In this study, most patients and all those who followed after 1996 had annual pituitary scans for the first 5 years followed by 2 yearly imaging. All pituitary scans were reviewed by a single, experienced neuroradiologist in the pituitary multidisciplinary meetings at variable time intervals during the follow-up. For this analysis, the post-operative pituitary MRI at 3 months were classified as i) no residual tumour if there was no residual tumour or empty sella, ii) intrasellar if the residual (remnant) tumour was confined to the sella and iii) extrasellar if the residual tumour extended outside the sella into the suprasellar cistern or invaded the surrounding structures such as the cavernous or sphenoid sinuses. If the initial postoperative scans were missing or did not fit into the above classification, these were grouped under 'unclassified'. Tumour re-growth was defined as an increase in size of residual tumour compared with the previous postoperative follow-up scans. Recurrence was defined as the appearance of the tumour mass in a patient without residual tumour mass on the post-operative scans. The duration of observation was calculated from the date of surgery to the time of tumour re-growth/recurrence or to the last scan. One patient had repeat surgery 15 months after the first pituitary surgery due to the presence of significant residual tumour and the followup period was calculated from the second surgery to the last pituitary scan.

Ophthalmological evaluation included assessment of the visual acuity and visual fields, performed by a Humphrey or Goldmann perimeter before surgery, in the immediate post-operative period $(<1$ week in most cases) and at 12 weeks (in most cases) after surgery. All patients underwent pituitary function tests before and 6 weeks post-operatively. Endocrine results are not reported in this study.

\section{Statistical analysis}

Data are expressed as mean ( \pm s.D.) when parametric and as median (range) when non-parametric. The association between each baseline characteristic and changes in the residual tumour size were examined using Cox regression analysis. The Cox multivariate regression analysis and life table analysis (Breslow (Wilcoxon) and Wilcoxon (Gehan) tests) were used to assess the relative risk of pituitary residual tumour characteristics for tumour re-growth against time. Tumour re-growth-free survival was estimated by

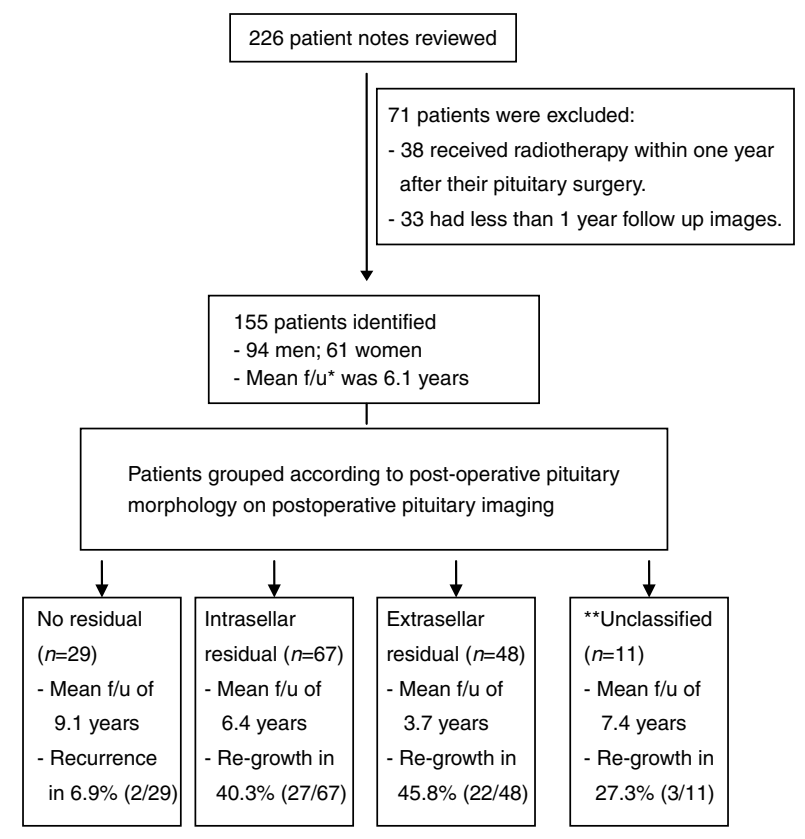

Figure 1 Flow chart of subjects studied. ${ }^{*}$ /u, follow-up; ** patients in whom tumour morphology could not be classified into any of the groups or where the initial post-operative scans were missing. 
Table 1 Relationship between baseline characteristics (pre- and post-operative) and pituitary tumour recurrence/re-growth. Results of the Cox multivariate regression analysis are as above. Patients in whom tumour morphology could not be classified into any of the groups or where the initial post-operative scans were missing were excluded from analysis.

\begin{tabular}{|c|c|c|c|c|}
\hline & $\begin{array}{l}\text { Re-growth } \\
(n=51)\end{array}$ & $\begin{array}{l}\text { No re-growth } \\
\quad(n=93)\end{array}$ & $P$ value & $\begin{array}{l}\text { Hazard } \\
(95 \% \mathrm{Cl})\end{array}$ \\
\hline \multicolumn{5}{|l|}{$\operatorname{Sex}(\%)$} \\
\hline Female $(n=55)$ & $17(36.2)$ & $38(63.8)$ & 0.620 & $1.204(0.578-2.506)$ \\
\hline Male $(n=89)$ & $34(32.8)$ & 55 (67.2) & & \\
\hline \multicolumn{5}{|l|}{ Age at surgery (years) } \\
\hline Mean (S.D.) & $53.7(13.4)$ & $60.7(14.4)$ & 0.034 & $0.975(0.974-0.998)$ \\
\hline Median (range) & $53.2(22.3-83.7)$ & $62.6(18.3-88)$ & & \\
\hline \multicolumn{5}{|c|}{ Immunohistochemistry (\%) } \\
\hline ACTH negative & $46(35)$ & $82(65)$ & 0.454 & $1.492(0.523-4.256)$ \\
\hline \multirow{2}{*}{\multicolumn{5}{|c|}{ Tumour characteristics (\%) }} \\
\hline & & & & \\
\hline No residual tumour & $2(6.9)$ & $27(93.1)$ & $<0.001$ & $0.035(0.008-0.159)$ \\
\hline Intrasellar residual & $27(40.3)$ & $40(59.7)$ & 0.001 & $0.323(0.161-0.647)$ \\
\hline Extrasellar residual $^{\mathrm{C}}$ & $22(45.8)$ & $26(54.2)$ & & \\
\hline IS + Cav & $14(45.1)$ & $17(54.9)$ & $\mathrm{N} / \mathrm{A}$ & - \\
\hline $\mathrm{SS}+\mathrm{Cav}$ & $4(50)$ & $4(50)$ & $\mathrm{N} / \mathrm{A}$ & - \\
\hline SS only & $4(50)$ & $4(50)$ & $\mathrm{N} / \mathrm{A}$ & - \\
\hline
\end{tabular}

N, total number; IS, intrasellar; SS, suprasellar; Cav, cavernous sinus involvement; $95 \% \mathrm{Cl}, 95 \%$ confidence interval; N/A, not analysed.

The regression coefficients for the histochemistry categories are relative to the reference category, which is ACTH positive (silent).

${ }^{\mathrm{b}}$ Characteristics of residual pituitary morphology based on post-operative imaging. The regression coefficients for the tumour characteristics are relative to the reference category, which is extrasellar residual.

${ }^{\mathrm{c}}$ Extrasellar residual group included those with IS + Cav, SS + Cav and SS only.

using Kaplan-Meier method. $P<0.05$ was considered statistically significant. SPSS Software (version 19.0; SPSS Inc., Chicago, IL, USA) was used.

\section{Results}

One hundred and fifty-five patients (94 males, mean age 57.9 (range 18.3-88) years) fulfilled the criteria. Ninety-nine per cent of patients $(n=154)$ had undergone transsphenoidal pituitary resection and the remaining patients were operated via the trans-cranial route.

The mean observation period following surgery was 6.1 years (s.D. 4.9) and the median was 4.3 years (range 1-25.8). The first (post-operative) scan occurred at a median of 3 months (interquartile range (IQR) 2 months). The second scan occurred at a median of 15 months (IQR 7 months) from the surgery. Eighty per cent of the patients had their imaging according to our protocol at the scheduled time frame \pm 7 months in $80 \%$ of the times. Overall re-growth was documented in $54(34.8 \%)$ cases and $20.4 \%(11 / 54)$ of these showed relapse/re-growth 10 or more years after the initial surgery. Post-operatively, pituitary imaging showed no evidence of remnant mass in $29(18.7 \%)$ patients, intrasellar only remnant in $67(43.2 \%)$ patients and extrasellar remnant in 48 (31\%) patients. Eleven $(7.1 \%)$ patients were grouped under 'unclassified' and were not included in the analysis. Figure 1 shows the study population. The pre-operative and post-operative findings in the patients in the study analysis are shown in Table 1.

The re-growths were $6.9 \%(2 / 29), 40.3 \%(27 / 67)$ and $45.8 \%(22 / 48)$ in those who had no residual tumour, intrasellar remnant only and extrasellar remnant on their post-operative pituitary scans respectively. Amongst the 'unclassified', re-growth was observed in $27.3 \%(3 / 11)$.

Kaplan-Meier analysis showed relapse rates of 23.1, 46.7 and $67.9 \%$ at 5, 10 and 15 years respectively. Fifty per cent of the re-growths were detected in 7 years, $75 \%$ in 9.8 years and $95 \%$ in 17 years (range $1-25.8$ ). Re-growth rates were different between the groups based on post-operative tumour characteristics. Relapse

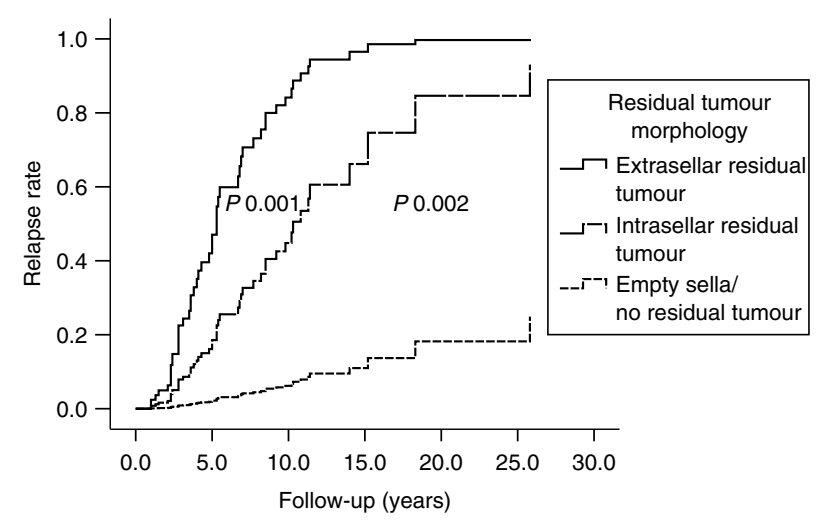

Figure 2 Relapse rate within three subgroups of patients according to post-operative scan classification. 
Table 2 Re-growth rates detected by 1, 5 and 10 years in the three groups based on post-operative imaging details.

\begin{tabular}{lccc}
\hline & $\begin{array}{c}\text { No residual } \\
\text { tumour (A) }\end{array}$ & $\begin{array}{c}\text { Intrasellar } \\
\text { residual (B) }\end{array}$ & $\begin{array}{c}\text { Extrasellar } \\
\text { residual (C) }\end{array}$ \\
\hline At 1 year (\%) & 0 & 1.5 & 4.2 \\
At 5 years (\%) & 0 & 20 & 53 \\
At 10 years (\%) & 6 & 53 & 80 \\
$P$ value & & 0.001 & $<0.001$ \\
Versus A & - & - & $<0.001$ \\
Versus B & 0.001 & $<0.001$ & - \\
Versus C & $<0.001$ & & \\
\hline
\end{tabular}

rate at 5 years was $53 \%$ in those with a post-operative extrasellar tumour residual compared with $20 \%$ in patients with an intrasellar tumour post-operatively. No patient relapsed in the first 5 years in the group that had no post-operative tumour detected on the immediate scan post-operatively. Furthermore, mean time to detection of re-growth was $7.3 \pm 4.1$ years (median 6.8 years, range 1.3-18.3) and $3.5 \pm 2.5$ years (median 2.8 years, range 1-10.8) in patients with intrasellar remnant and extrasellar remnant respectively $(P \leq 0.001)$. Thus, by 5 years, $41.3 \%$ of the re-growths in the intrasellar remnant group occurred compared with $81.8 \%$ of the re-growths in those with an extrasellar residual tumour. Furthermore, in subjects with extrasellar remnant, mean time to re-growth was $3.34 \pm 2.1$ years for those with cavernous sinus invasion compared to $5.3 \pm 3.1$ years in those without cavernous sinus remnant.

Multivariate Cox regression analysis identified the post-operative MRI pituitary appearance as key independent risk factors for tumour re-growth (Table 1, Fig. 1). The presence of a tumour remnant postoperatively was strongly associated with the risk of re-growth $(P<0.001)$. There were no statistical differences in re-growths according to sex of the patient, but there was a higher re-growth rate in patients who were younger at the time of surgery $(P=0.034)$. Re-growth rate was also similar between tumours that stained positive and those stained negative for ACTH.

Figure 2 and Table 2 shows the re-growth-free survival rates for the three subgroups of patients (no residual tumour, intrasellar remnant only or extrasellar remnant). Figure 3 demonstrates the individual re-growths vs time and shows no fall off with time. Fifty per cent occurred in the first 5 years, $80 \%$ occurred in the first 10 years but $20 \%$ of re-growths occurred after 10 years.

\section{Discussion}

It is currently unclear for how long post-operative radiological surveillance is necessary in patients who have had an NFA operated on and in whom it is deemed that no RT is initially necessary. We have a large number of patients in this group who we have followed up systematically over a period of time up to 26 years. Further, we have a standard protocol of yearly scans for the first 5 years and 2 yearly scans thereafter. It is therefore clear that recurrent growth post-operatively is unlikely to be missed. We have found significant number of patients whose tumours have re-grown for the first time over 10 years post-operatively. Our study showed that there was no tail off of recurrent growth over this period of time. It seems therefore that postoperative surveillance of non-functioning tumours needs to be continued for long term.

There are other conclusions to this study. Our analysis shows that the size of the remnant postoperatively predicts the relapse rate. Relapse rate at 5 years was $53 \%$ in those with a post-operative extrasellar tumour residual compared with $20 \%$ in patients with an intrasellar tumour post-operatively. Interestingly, no patients relapsed in the first 5 years in the group that had no post-operative tumour detected on the immediate scan post-operatively. Furthermore, the time to re-growth was shorter for those with an extrasellar remnant post-operatively, $3.3 \pm 2.17$ years for those with a cavernous sinus remnant and $5.3 \pm 3.1$ years for those without cavernous involvement. There were only two recurrences observed in those who had no residual tumour on the post-operative scans, one at 5.3 years and the other at 25.8 years. Finally, patients who were younger at surgery showed a statistically significant increase in relapse. Relapse rates were not significantly different between those who had positive ACTH-staining pituitary tumours and nonACTH-staining pituitary tumours as seen in previous studies by Bradley et al. (18) and Cho et al. (19). As we stopped the follow-up after their first relapse, we did not study for any other aggressive features like multiple recurrences that were shown by these authors.

These data suggest that it may be worth considering irradiation for those patients who have post-operatively got a significant tumour remnant and in particular an extrasellar pituitary remnant. The duration of follow-up was clearly important, and with longer follow-up, the rates of re-growth increase.

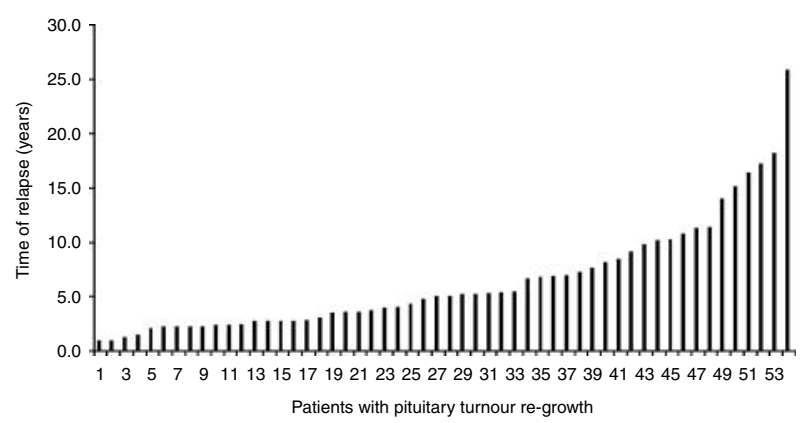

Figure 3 Histogram showing the timing (in years) of detection of tumour re-growth or recurrence in each individual within those patients who relapsed. 
Table 3 Studies showing calculated relapse-free survival at 10 years in NFA patients following surgery only.

\begin{tabular}{lrccc}
\hline Studies & NRT & $\begin{array}{c}\text { F/U } \\
\text { > } \mathbf{1 0} \text { years }^{\mathbf{a}}\end{array}$ & $\begin{array}{c}\text { Mean F/U years } \\
\text { (range) }\end{array}$ & $\begin{array}{c}\text { Calculated 10-year } \\
\text { RFS rates }(\%)\end{array}$ \\
\hline Turner et al. (7) & 65 & NA & $6.3(2-14)$ & 56 \\
Park et al. (13) & 132 & NA & $3.8(0.5-16.5)^{\mathrm{b}}$ & 49.5 \\
Dekkers et al. (8) & 91 & NA & $6 \pm 3.7(1-14)$ & 79 \\
Van den Bergh et al. (12) & 28 & NA & $5.9(0.25-17)$ & 22 \\
O'Sullivan et al. (11) & 126 & NA & $5.7(1-25.3)^{\mathrm{b}}$ & 49.5 \\
Gittoes et al. (14) & 63 & ?20 & $8.1(1-20)$ & 47 \\
Present study & 155 & 29 & $6.3 \pm 3.7(1-25.8)$ & 53.3 \\
\hline
\end{tabular}

NRT, number of patients who did not receive post-operative pituitary radiotherapy in each study; RFS, relapse-free survival; NA, not available.

${ }^{a}$ Number of patients followed up for more than 10 years following surgery.

${ }^{\mathrm{b}}$ Median.

Other studies have looked at relapse rates over time (Table 3), but our study reports follow-up of significant number of patients over 10 years. Greenman et al. (5) found similar re-growth rates to us and other studies like those of O'Sullivan et al. (11) and Soto-Ares et al. (9) did not find any re-growth in those with absent residual tumour/empty sellar post-operatively. However, in the study by O'Sullivan et al., the imaging protocol was not as rigorous as ours. There was imaging up to 5 years, but thereafter, it was up to the individual clinician's discretion. In the study by Soto-Ares et al., there were fewer number than in this study with a shorter duration of follow-up. In the study by Greenman et al. also, there was a shorter follow-up period than that reported in this study.

In terms of cost-effectiveness, it may be that as a result of this study, we do not need to keep such a close eye on patients in whom the post-operative scan does not show any residual tumour. We calculate 190 scans were performed in 29 patients with no post-operative tumour to detect two recurrences (one at 5.3 years and the other at 25.8 years). It seems clear that with the higher incidence of re-growth in those with post-operative intra- and extra-sellar tumour, the scanning regimen that we currently follow seems necessary.

This was a retrospective study with all the associated disadvantages of this. However, with a regular scanning protocol, we feel that we are unlikely to have missed recurrent tumour growth. We have attempted to look at histological markers of aggressive tumour behaviour, but on the data that we have looked at, we do not have sufficient number to assess the usefulness of these histological criteria such as MIB1 and atypia. Further, although we have not analysed this, the patients with markers of tumour aggressiveness may have been irradiated earlier.

\section{Conclusion}

In this large study of patients with NFA treated solely by surgery and followed up for an extended period, we have shown that a significant number of patients develop tumour re-growth for the first time more than 10 years after the surgery. We believe that patients with NFAs should currently be followed up long term and certainly for more than 10 years. The data herein do not give an indication as to when surveillance can be stopped. With the higher frequency of relapse that is earlier in those patients with extrasellar tumour on the post-operative scan, this may be an indication for early irradiation in this group. Younger patients need to be followed up more carefully and patients with no residual tumour post-operatively may possibly be followed up by imaging less frequently. It is unclear why some tumours appear to re-grow many years after initial surgery.

\section{Declaration of interest}

The authors declare that there is no conflict of interest that could be perceived as prejudicing the impartiality of the research reported.

\section{Funding}

This research did not receive any specific grant from any funding agency in the public, commercial or not-for-profit sector.

\section{References}

1 Daly AF, Rixhon M, Adam C, Dempegioti A, Tichomirowa MA \& Beckers A. High prevalence of pituitary adenomas: a crosssectional study in the province of Liege, Belgium. Journal of Clinical Endocrinology and Metabolism 200691 4769-4775. (doi:10. 1210/jc.2006-1668)

2 Davies JRE, Farrell WE \& Clayton RN. Pituitary tumours. Reproduction 2001121 363-371. (doi:10.1530/rep.0.1210363)

3 Karavitaki N, Collison K, Halliday J, Byrne JV, Price P, Cudlip S \& Wass JA. What is the natural history of nonoperated nonfunctioning pituitary adenomas? Clinical Endocrinology 200767 938-943. (doi:10.1111/j.1365-2265.2007.02990.x)

4 Bradley KM, Adams CB, Potter CP, Wheeler DW, Anslow PJ \& Burke CW. An audit of selected patients with non-functioning pituitary adenoma treated by transsphenoidal surgery without irradiation. Clinical Endocrinology 199441 655-659. (doi:10. 1111/j.1365-2265.1994.tb01832.x)

5 Greenman Y, Ouaknine G, Veshchev I, Reider-Groswasser II, Segev Y \& Stern N. Postoperative surveillance of clinically 
nonfunctioning pituitary macroadenomas: markers of tumour quiescence and re-growth. Clinical Endocrinology $2003 \mathbf{5 8}$ 763-769. (doi:10.1046/j.1365-2265.2003.01784.x)

6 Losa M, Mortini P, Barzaghi R, Ribotto P, Terreni MR, Marzoli SB, Pieralli S \& Giovanelli M. Early results of surgery in patients with nonfunctioning pituitary adenomas and analysis of the risk of tumor recurrence. Journal of Neurosurgery 2008108 525-532. (doi:10.3171/JNS/2008/108/3/0525)

7 Turner HE, Stratton IM, Byrne JV, Adams CB \& Wass JA. Audit of selected patients with nonfunctioning pituitary adenomas treated without irradiation-a follow-up study. Clinical Endocrinology 1999 51 281-284. (doi:10.1046/j.1365-2265.1999.00865.x)

8 Dekkers OM, Pereira AM, Roelfsema F, Voormolen JH, Neelis KJ, Schroijen MA, Smit JW \& Romijn JA. Observation alone after transsphenoidal surgery for non-functioning pituitary macroadenoma. Journal of Clinical Endocrinology and Metabolism 200691 1796-1801. (doi:10.1210/jc.2005-2552)

9 Soto-Ares G, Cortet-Rudelli C, Assaker R, Boulinguez A, Dubest C, Dewailly D \& Pruvo JP. MRI protocol technique in the optimal therapeutic strategy of non-functioning pituitary adenomas. European Journal of Endocrinology 2002146 179-186. (doi:10. 1530/eje.0.1460179)

10 Ferrante E, Ferraroni M, Castrignano T, Menicatti L, Anagni M, Reimondo G, Del Monte P, Bernasconi D, Loli P, Faustini-Fustini M, Borretta G, Terzolo M, Losa M, Morabito A, Spada A, BeckPeccoz P \& Lania AG. Non-functioning pituitary adenoma database: a useful resource to improve the clinical management of pituitary tumors. European Journal of Endocrinology 2006155 823-829. (doi:10.1530/eje.1.02298)

11 O'Sullivan EP, Woods C, Glynn N, Behan LA, Crowley R, O'Kelly P, Smith D, Thompson CJ \& Agha A. The natural history of surgically treated but radiotherapy-naïve nonfunctioning pituitary adenomas. Clinical Endocrinology 200971 709-714. (doi:10.1111/j. 1365-2265.2009.03583.x)

12 van den Bergh ACM, van den Bergh G, Schoorl MA, Sluiter WJ, van der Vliet AM, Hoving EW, Szabó BG, Langendijk JA, Wolffenbuttel BH \& Dullaart RP. Immediate postoperative radiotherapy in residual non-functioning pituitary adenoma: beneficial effect on local control without additional negative impact on pituitary function and life expectancy. International Journal of Radiation Oncology 200767 863-869. (doi:10.1016/j.ijrobp. 2006.09.049)

13 Park P, Chandler WF, Barkan AL, Orrego JJ, Cowan JA, Griffith KA $\&$ Tsien $\mathrm{C}$. The role of radiation therapy after surgical resection of nonfunctional pituitary macroadenomas. Neurosurgery 200455 100-106. (doi:10.1227/01.NEU.0000126885.71242.D7)

14 Gittoes NJ, Bates AS, Tse W, Bullivant B, Sheppard MC, Clayton RN \& Stewart PM. Radiotherapy for non-function pituitary tumours. Clinical Endocrinology 199848 331-337. (doi:10.1046/j.13652265.1998.00393.x)

15 Gittoes NJL. Radiotherapy for non-functioning pituitary tumors when and under what circumstances? Pituitary 20036 103-108. (doi:10.1023/B:PITU.0000004801.95086.e2)

16 Erridge SC, Conkey DS, Stockton D, Strachan MW, Statham PF, Whittle IR, Grant R, Kerr GR \& Gregor A. Radiotherapy for pituitary adenomas: long-term efficacy and toxicity. Radiotherapy and Oncology 200993 597-601. (doi:10.1016/j.radonc.2009. 09.011)

17 Woollons AC, Hunn MK, Rajapakse YR, Toomath R, Hamilton DA, Conaglen JV \& Balakrishnan V. Nonfunctioning pituitary adenomas: indications for postoperative radiotherapy. Clinical Endocrinology 200053 713-717. (doi:10.1046/j.1365-2265. 2000.01153.x)

18 Bradley KJ, Wass JA \& Turner HE. Non-functioning pituitary adenomas with positive immunoreactivity for ACTH behave more aggressively than ACTH immunonegative tumours but do not recur more frequently. Clinical Endocrinology 200358 59-64. (doi:10.1046/j.1365-2265.2003.01674.x)

19 Cho HY, Cho SW, Kim SW, Shin CS, Park KS \& Kim SY. Silent corticotroph adenomas have unique recurrence characteristics compared with other nonfunctioning pituitary adenomas. Clinical Endocrinology 201072 648-653. (doi:10.1111/j.1365-2265. 2009.03673.x)

Received 26 June 2011

Revised version received 4 August 2011

Accepted 7 September 2011 